EPJ Web of Conferences 20, 04001 (2012)

DOI: $10.1051 /$ epjconf/20122004001

(C) Owned by the authors, published by EDP Sciences, 2012

\title{
Effect of exotic long-lived sub-strongly interacting massive particles in big bang nucleosynthesis and a new solution to the Li problem
}

\author{
Masahiro Kawasaki ${ }^{1,2}$ and Motohiko Kusakabe ${ }^{1}$ \\ 1 Institute for Cosmic Ray Research, University of Tokyo, Kashiwa, Chiba 277-8582, Japan e-mail: \\ kusakabe@icrr.u-tokyo.ac.jp \\ 2 Institute for the Physics and Mathematics of the Universe, University of Tokyo, Kashiwa, Chiba \\ 277-8582, Japan
}

\begin{abstract}
The plateau of ${ }^{7} \mathrm{Li}$ abundance as a function of the iron abundance by spectroscopic observations of metal-poor halo stars (MPHSs) indicates its primordial origin. The observed abundance levels are about a factor of three smaller than the primordial ${ }^{7} \mathrm{Li}$ abundance predicted in the standard Big Bang Nucleosynthesis (BBN) model. This discrepancy might originate from exotic particle and nuclear processes operating in BBN epoch. Some particle models include heavy $(m \gg 1 \mathrm{GeV})$ long-lived colored particles which would be confined inside exotic heavy hadrons, i.e., strongly interacting massive particles (SIMPs). We have found reactions which destroy ${ }^{7} \mathrm{Be}$ and ${ }^{7} \mathrm{Li}$ during $\mathrm{BBN}$ in the scenario of BBN catalyzed by a long-lived sub-strongly interacting massive particle (sub-SIMP, $X$ ). The reactions are non radiative $X$ captures of ${ }^{7} \mathrm{Be}$ and ${ }^{7} \mathrm{Li}$ which can be operative if the $X$ particle interacts with nuclei strongly enough to drive ${ }^{7} \mathrm{Be}$ destruction but not strongly enough to form a bound state with ${ }^{4} \mathrm{He}$ of relative angular momentum $L=1$. We suggest that ${ }^{7} \mathrm{Li}$ problem can be solved as a result of a new process beyond the standard model through which the observable signature was left on the primordial Li abundance.
\end{abstract}

\section{Introduction}

The standard big bang nucleosynthesis (BBN) model predicts primordial light element abundances considerably consistent with abundances inferred from astronomical observations. However, there is a discrepancy in ${ }^{7} \mathrm{Li}$ abundances predicted in the standard BBN (SBBN) model and inferred from observations [1,2]. Primordial lithium abundances are inferred from measurements in metal-poor halo stars (MPHSs). Observed abundances are roughly constant as a function of metallicity [3,4,1,2,5-7] at ${ }^{7} \mathrm{Li} / \mathrm{H}=(1-2) \times 10^{-10}$. The theoretical prediction of the SBBN model is, however, a factor of 2-4 higher, e.g., ${ }^{7} \mathrm{Li} / \mathrm{H}=\left(5.24_{-0.67}^{+0.71}\right) \times 10^{-10}$ [8], when its parameter, the baryon-to-photon ratio $\eta$, determined from the observation with Wilkinson Microwave Anisotropy Probe (WMAP) of the cosmic microwave background (CMB) radiation [9] is used. This discrepancy may be caused by some astrophysical process to reduce ${ }^{7} \mathrm{Li}$ abundances in stellar surfaces. The gravitational settling in the model including a combination of the atomic and turbulent diffusion $[10,11]$ provides a possible explanation of the Li discrepancy. The precise trend of $\mathrm{Li}$ abundance as a function of effective temperature of stars in the metal-poor globular cluster NGC 6397 is, however, not reproduced theoretically [12]. Thus, a realistic astrophysical solution is not obtained yet.

Some particle models beyond the standard model include heavy ( $m \gg 1 \mathrm{GeV}$ ) long-lived colored particles. The scenarios include split supersymmetry [13,14], weak scale supersymmetry with a longlived gluino [15-17] or squark [18] as the next-to-lightest supersymmetric particles, and extended theories with new kinds of colored particles $[19,20]$. The long-lived heavy colored particles would be

This is an Open Access article distributed under the terms of the Creative Commons Attribution-Noncommercial License 3.0, which permits unrestricted use, distribution, and reproduction in any noncommercial medium, provided the original work is properly cited. 


\section{EPJ Web of Conferences}

confined at temperatures below the deconfinement temperature $T_{C} \sim 180 \mathrm{MeV}$ inside exotic heavy hadrons, i.e., strongly interacting massive particles (SIMPs) which we call $X$ particles [21].

If the annihilation cross section is a typical value for strong interaction, i.e., $\sigma \sim O\left(\mathrm{GeV}^{-1}\right)^{2}$ the final abundance of $X$ particles is estimated assuming that their abundances are fixed when the annihilation rate becomes smaller than the Hubble expansion rate of the universe [21]. The relic abundance is then given by

$$
\frac{N_{X}}{n_{b}} \sim 0.5 \times 10^{-8}\left(\frac{g_{*}}{10.75}\right)^{1 / 2}\left(\frac{m}{\mathrm{TeV}}\right)^{1 / 2}\left(\frac{T_{B}}{180 \mathrm{MeV}}\right)^{-3 / 2}\left(\frac{\sigma}{m_{\pi}^{-2}}\right)^{-1},
$$

where $N_{X}$ is the number density of the $X$ particle, $n_{b}$ is the number density of baryons, $g_{*}$ is the total number of effective massless degrees of freedom in terms of number [22], $m$ is the mass ( $m \gg$ $1 \mathrm{GeV}$ ) of the heavy long-lived colored particles, $T_{B}$ is the temperature of the universe at which the $X$-particles are formed, $\sigma$ is the annihilation cross section of the $X$ particle, and $m_{\pi} \sim 140 \mathrm{MeV}$ is the mass of pion. The thermal relic abundance depends on the annihilation cross section which is not known. A nonthermal production of long-lived colored particles might have added after the freezeout of annihilation to the freezeout abundance. We then consider the $X$ abundance as a free parameter in this paper taking account of such uncertainties.

Observational constraints on hypothetical SIMPs have been studied [23-25]. Effects of exotic neutral stable hadrons on BBN were studied in Ref. [26]. The strong force between a nucleon and a exotic hadron $(X)$ is assumed to be similar to that between a nucleon $N$ and a $\Lambda$ hyperon. Another assumption is that new hadrons are captured in a bound state of ${ }^{4} \mathrm{He}$ plus $X$ after BBN. They then suggested that beryllium has the largest number fraction $A_{X} / A$ of bound states with the hadrons among the light elements produced in BBN, where the $A$ and $A_{X}$ are a nuclide $A$ and a bound state of $A$ with a hadron $X$. Mohapatra and Teplitz [27] estimated the cross section of $X$ capture by ${ }^{4} \mathrm{He}$, and suggested that a large fraction of free $X$ particles would not become bound into light nuclides and remain free contrary to the previous suggestion [26]. In both of the two studies, it has been assumed that exotic hadrons interact with normal nuclei by typical strengths of strong interaction and implicitly assumed that its mass is about that of $\Lambda$ hyperon, i.e., $m_{X} \sim 1.116 \mathrm{GeV}$ [28].

Effects on BBN of long-lived exotic hadrons of $m \gg 1 \mathrm{GeV}$, such as a weak-scale mass, have been studied [29]. The interaction strength between an $X$ particle and a nucleon is assumed to be similar to that between nucleons. Rates of many reactions involving the $X$ particle were estimated, and a network calculation of the nucleosynthesis including effects of the $X$ was performed. The constraint on the decay lifetime of such $X$ particles, i.e., $\tau_{X} \lesssim 200 \mathrm{~s}$ was then derived from a comparison of calculated abundances with observational abundance constraints.

The model [29] has two interesting predictions, i.e., signatures of the $X$ particles on primordial abundances to be seen in future astronomical observations: 1) ${ }^{9} \mathrm{Be}$ and $\mathrm{B}$ can be produced in amounts more than predicted in the SBBN. 2) The isotopic ratio ${ }^{10} \mathrm{~B} /{ }^{11} \mathrm{~B}$ tends to be very high. This is different from predictions of other standard models for boron production, i.e., the cosmic ray nucleosynthesis $\left({ }^{10} \mathrm{~B} /{ }^{11} \mathrm{~B} \sim 0.4[30-32]\right)$ or the supernova neutrino process $\left({ }^{10} \mathrm{~B} /{ }^{11} \mathrm{~B} \ll 1[33,34]\right)$. They did not find a solution to the ${ }^{6} \mathrm{Li}$ or ${ }^{7} \mathrm{Li}$ problems under their assumption.

The strength of interaction between long-lived exotic hadrons $X$ and a nucleon is not known. Their masses are also unknown. We, therefore, investigate effects of such particles in various cases of interaction strengths and masses. We found then a new possibility that reactions associated with the $X$ particle reduce ${ }^{7} \mathrm{Be}$ abundance and that the ${ }^{7} \mathrm{Li}$ problem is solved.

In this paper we show the destruction mechanism of ${ }^{7} \mathrm{Be}$ in the presence of the $X$ particle. We perform a network calculation of BBN in the presence of a long-lived SIMP $X^{0}$ of a zero charge taking the mass and the strength of interaction with a nucleon as characterizing parameters. In Sec. 2 assumptions on the $X^{0}$ particle and estimations for binding energies between nuclei and an $X^{0}$ are described (see Ref. [35] for our estimation for rates of important reactions). In Sec. 3 the destruction processes of ${ }^{7} \mathrm{Be}$ and ${ }^{7} \mathrm{Li}$ are described. With results of the network calculations of BBN, we delineate the parameter region in which the ${ }^{7} \mathrm{Be}$ and ${ }^{7} \mathrm{Li}$ destructions possibly operate. In Sec 4 we summarize conclusions of this work.

In this paper, we use the notation, i.e., $1(2,3) 4$ for a reaction $1+2 \rightarrow 3+4$. 


\section{Model}

The existence of a strongly interacting massive particle (SIMP) $X$ during the BBN epoch is assumed. The spin is zero and charge is also zero. The mass is one parameter. Two types of nuclear potentials between an $X^{0}$ and a nucleon $(X N)$ are considered in Ref. [35]. In this paper we only consider the Gaussian $X N$ potential given by

$$
v(r)=v_{0} \delta \exp \left[-\left(r / r_{0}\right)^{2}\right],
$$

where $v_{0}=-72.15 \mathrm{MeV}$ and $r_{0}=1.484 \mathrm{fm}$, and the interaction strength is varied by changing $\delta$, the second parameter. When the $\delta$ equals unity then the binding energy of deuteron, i.e., $2.224 \mathrm{MeV}$ is derived.

The potential between an $X^{0}$ and a nuclide $A(X A)$ is given by

$$
V(r)=\int v(\boldsymbol{x}) \rho\left(\boldsymbol{r}^{\prime}\right) d \boldsymbol{r}^{\prime}
$$

where $\boldsymbol{r}$ is the radius from an $X$ to the center of mass of $A, \boldsymbol{r}^{\prime}$ is the distance between the center of mass of $A$ and a nucleon inside the nuclide $A, \boldsymbol{x}=\boldsymbol{r}+\boldsymbol{r}^{\prime}$ is the distance between the $X$ and the nucleon, and $\rho\left(\boldsymbol{r}^{\prime}\right)$ is the nucleon density of the nucleus which is generally distorted by potential of an $X^{0}$ from the density of normal nucleus [36]. Under the assumption of spherical symmetry in nucleon density, i.e., $\rho(r)$, the potential is written in the form of

$$
V(r)=\pi v_{0} \delta \frac{r_{0}^{2}}{r} \int_{0}^{\infty} d r^{\prime} r^{\prime} \rho\left(r^{\prime}\right)\left\{\exp \left[-\frac{\left(r-r^{\prime}\right)^{2}}{r_{0}^{2}}\right]-\exp \left[-\frac{\left(r+r^{\prime}\right)^{2}}{r_{0}^{2}}\right]\right\}
$$

As a crude assumption, the nucleon density $\rho(r)$ is approximately given by the undistorted one for normal nucleus. The folding of $X N$ potentials to derive $X A$ potential [equation (3)] does not exactly output the real $X A$ potential. In order to derive precise results of nuclear structures or energy levels, many-body models for an $X^{0}$ particle and nuclear clusters composing the nucleus should be used as were done in the case of hypernuclei [37].

The nucleon density of nuclei with mass number $A \geq 2$ is assumed to be Gaussian, i.e,

$$
\rho(r)=\rho(0) \exp \left[-(r / b)^{2}\right]
$$

where $\rho(0)=A \pi^{-3 / 2} b^{-3}$ is the nucleon density at $r=0$ and satisfies the normalization $\int \rho(r) d \boldsymbol{r}=A$, with $A$ the mass number. The parameter for the width of density, i.e., $b$, is related to the root mean square (RMS) nuclear matter radius determined from experiments, i.e., $b=\sqrt{2 / 3} r_{\mathrm{m}}^{\mathrm{RMS}}$.

Our estimation for rates of nonradiative and radiative nuclear reactions and $\beta$-decay as well as our nuclear reaction network are explained in detail in Ref. [35].

\subsection{Nuclear Binding Energies}

The BBN catalyzed by the $X$ particle is significantly sensitive to binding energies of nuclei to an $X^{0}$ particle ( $X$-nuclei). The binding to $X$ particles changes the relative energies of initial and final states, and may even change the sign of the $Q$-value. [29]. We calculate binding energies and eigenstate wave functions of $X$-nuclei. We solve the two-body Shrödinger equation by a variational calculation using the Gaussian expansion method [38], and obtain binding energies. We assumed the spherical symmetry in the nuclear potential.

If the mass of the $X^{0}$ particle, i.e., $m_{X}$, is much heavier than light nuclides, the reduced mass $\mu$ approaches asymptotically to the mass of the nuclide. The binding energies, therefore, also approach to asymptotic values in the limit of large $m_{X}$.

The adopted RMS nuclear matter radii are listed in Table 1 of Ref. [35]. The interaction strength $\delta$ and the mass $m_{X}$ of the $X$ particle are taken as parameters. Binding energies of ground state $X$-nuclei are calculated. Using the obtained binding energies, $Q$-values of various reactions are calculated. We 


\section{EPJ Web of Conferences}

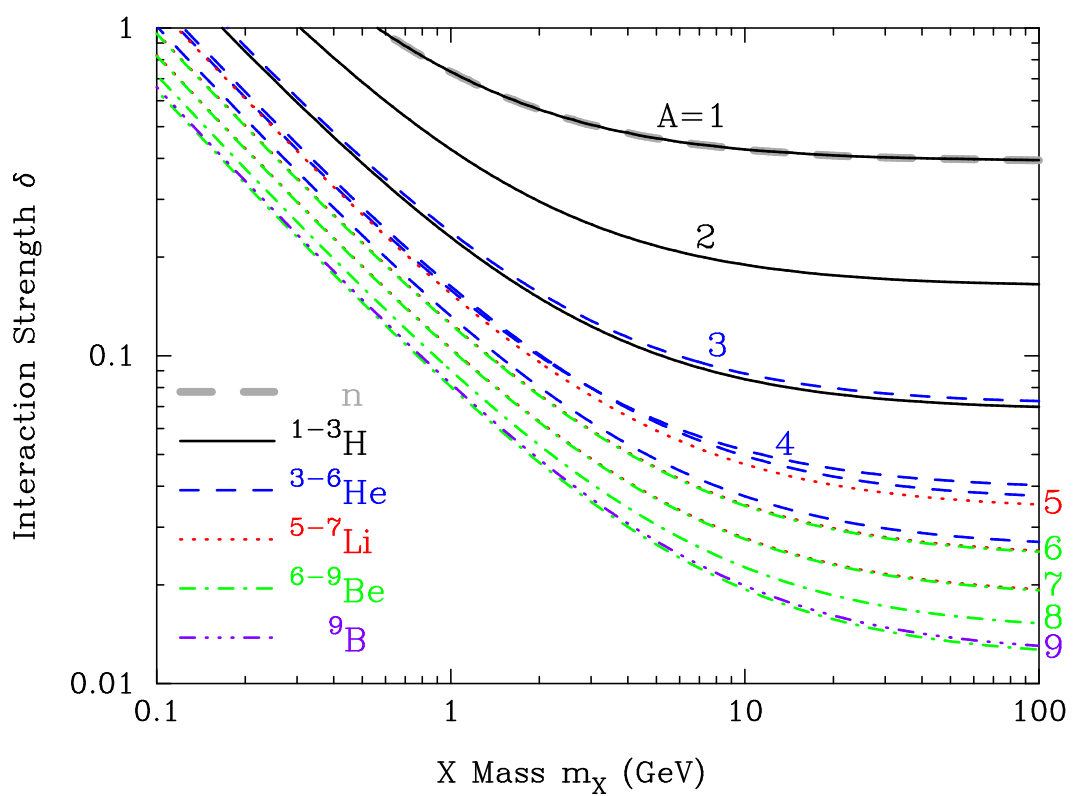

Fig. 1. Contours of binding energies between nuclei and an $X^{0}$ corresponding to $0.1 \mathrm{MeV}$ for the case of the Gaussian $X N$ potential. Numbers attached to the contours indicate mass numbers of nuclei. This figure is reprinted from Ref. [35]

also calculate binding energies of nuclear excited states of ${ }^{4} \mathrm{He}_{X}^{*}$ and ${ }^{8} \mathrm{Be}_{X}^{*}$ with relative angular momentum of $L=1$.

Figure 1 shows the contours of binding energies of ground state $X$-nuclei of mass numbers $A=1$ to 9 in the case of the Gaussian type $X N$ potential. The contours correspond to binding energy (BE) of $\mathrm{BE}=0.1 \mathrm{MeV}$. Since weakly bound $X$-nuclei of $\mathrm{BE} \lesssim O(0.1 \mathrm{MeV})$ tend to be photodisintegrated by background radiations, they do not work as effective paths for heavy element synthesis. In a parameter region located at right upper side from a contour, the $X$-nucleus can form during BBN epoch, and thus possibly affects BBN.

\section{Results}

Figure 2 shows results of the abundances of normal and $X$-nuclei as a function of temperature, i.e., $T_{9} \equiv T /\left(10^{9} \mathrm{~K}\right)$, for $\delta=0.1$. The mass of the $X^{0}$ was set to be $m_{X}=100 \mathrm{GeV}$. The initial abundance of the $X$ is $N_{X} / n_{b}=1.7 \times 10^{-4}\left(Y_{X} \equiv N_{X} / s=1.5 \times 10^{-14}\right)$, where $N_{X}$ and $n_{b}$ are the number densities of the $X^{0}$ particles and baryons, respectively ( $s$ is the entropy density). The decay lifetime is assumed to be much longer than BBN time scale, i.e., $\tau_{X} \gg 200 \mathrm{~s}$. We note that the $X^{0}$ particle is assumed to have been long extinct by now.

At high temperatures $T_{9} \gtrsim 1$, the $X^{0}$ particles are in the free state since efficient photodisintegrations of $X$-nuclei destroy the bound state. At $T_{9} \sim 1$ the ${ }^{4} \mathrm{He}$ synthesis occurs as in SBBN, and about one third of $X^{0}$ particles are captured by ${ }^{4} \mathrm{He}$ nuclei. ${ }^{4} \mathrm{He}_{X}$ nuclei produced in this epoch react with normal nuclei, and affect abundances of ${ }^{7} \mathrm{Li}\left[\right.$ by $\left.{ }^{4} \mathrm{He}{ }_{X}\left(t,{ }^{7} \mathrm{Li}\right) X\right],{ }^{6} \mathrm{Li}_{X}\left[\right.$ by $\left.{ }^{4} \mathrm{He}_{X}(d, \gamma){ }^{6} \mathrm{Li}_{X}\right]$, and ${ }^{8} \mathrm{Be}_{X}$ [by ${ }^{4} \mathrm{He}_{X}(\alpha, \gamma){ }^{8} \mathrm{Be}_{X}$ ]. Note that ${ }^{6} \mathrm{Li}_{X}$ nuclei produced at $T_{9} \sim 1$ experience a strong destruction process, i.e., ${ }^{6} \mathrm{Li}\left(p,{ }^{3} \mathrm{He} \alpha\right) X .{ }^{9} \mathrm{Be}_{X}$ is produced by ${ }^{8} \mathrm{Be}_{X}(d, p){ }^{9} \mathrm{Be}_{X}$. At last, the most important processes operate. Free $X^{0}$ particles which survived the capture by ${ }^{4} \mathrm{He}$ react with ${ }^{7} \mathrm{Be}\left[\right.$ by $\left.X\left({ }^{7} \mathrm{Be},{ }^{3} \mathrm{He}\right){ }^{4} \mathrm{He}_{X}\right]$ and ${ }^{7} \mathrm{Li}\left[\right.$ by $\left.X\left({ }^{7} \mathrm{Li}, t\right){ }^{4} \mathrm{He}_{X}\right]$. The abundances of ${ }^{7} \mathrm{Be}$ and ${ }^{7} \mathrm{Li}$ thus decrease.

Figure 3 shows abundances (solid curves) of ${ }^{4} \mathrm{He}$ (mass fraction), D, ${ }^{3} \mathrm{He},{ }^{7} \mathrm{Li}$ and ${ }^{6} \mathrm{Li}$ (by number relative to $\mathrm{H}$ ) as a function of the baryon-to-photon ratio $\eta$ or the baryon energy density $\Omega_{B} h^{2}$ 


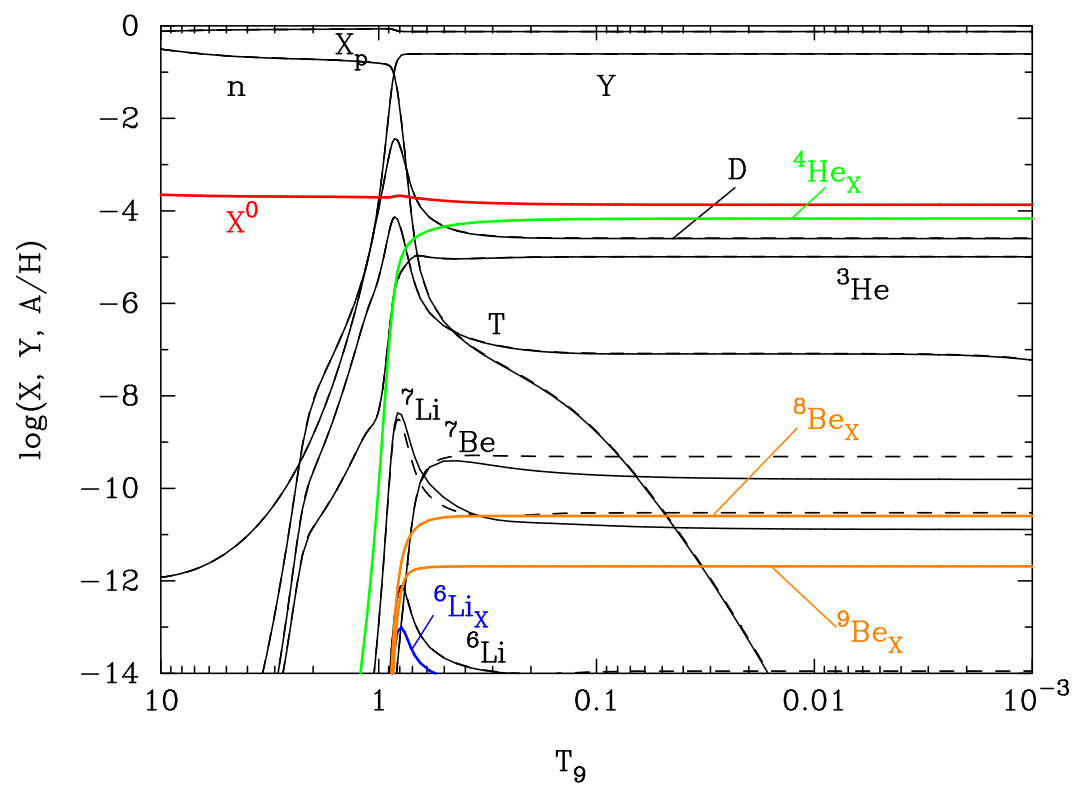

Fig. 2. Calculated abundances of normal and $X$-nuclei (solid lines) as a function of $T_{9}$. The mass of $X^{0}$ particle was assumed to be $m_{X}=100 \mathrm{GeV}$, and the interaction strength of $X N$ force is 0.1 times that of $N N$ force $(\delta=0.1)$. For this figure, we took the $X^{0}$ abundance to be $N_{X} / n_{b}=1.7 \times 10^{-4}\left(Y_{X} \equiv N_{X} / s=1.5 \times 10^{-14}\right)$, and its lifetime to be much longer than BBN time scale, i.e., $\tau_{X} \gg 200 \mathrm{~s}$. The $X^{0}$ reaction rates are given as described in Ref. [35]. The dashed lines correspond to the abundances of normal nuclei in the standard BBN model. This figure is reprinted from Ref. [35]

of the universe. The solid curves are the calculated result in this $X$ catalyzed BBN for $\left(m_{X}, \delta, Y_{X}\right.$, $\left.\tau_{X}\right)=\left(100 \mathrm{GeV}, 0.1,1.5 \times 10^{-14}, \infty\right)$. The dashed curves are those in the SBBN. The boxes correspond to the adopted constraints on primordial abundances [35]. The vertical stripe is the $2 \sigma$ limits on $\Omega_{\mathrm{B}} h^{2}=0.02258_{-0.00056}^{+0.00057}$ provided by WMAP [9] for the $\Lambda \mathrm{CDM}+\mathrm{SZ}+$ lens model.

The decrease in the ${ }^{7} \mathrm{Li}$ abundance is found, while other nuclear abundances are not changed. A solution to the ${ }^{7} \mathrm{Li}$ problem is thus found in this model.

If the strength of $X N$ interaction is relatively weak, most strong reactions for the $X^{0}$ particle to get bound to nuclei are non-radiative $X^{0}$-capture reactions which are found important in the present model. $X\left({ }^{6} \mathrm{Li}, d\right){ }^{4} \mathrm{He}_{X}, X\left({ }^{7} \mathrm{Li}, t\right)^{4} \mathrm{He}_{X}$, and $X\left({ }^{7} \mathrm{Be},{ }^{3} \mathrm{He}\right){ }^{4} \mathrm{He}_{X}$ are important reactions in this $X$ catalyzed BBN scenario.

Figure 4 shows contours in the parameter space $\left(m_{X}, \delta\right)$ for critical binding energies of $X$-nuclei (thin and thick smooth curves). Above the contours, $Q$-values for reactions [35] are positive. Zigzag curves show boundaries above which an excited state of ${ }^{4} \mathrm{He}^{*}$ with $L=1$ (upper line) and ${ }^{8} \mathrm{Be}^{*}$ with $L=1$ (lower) exist, respectively. When there is the excited state ${ }^{4} \mathrm{He}^{*}(L=1)$, the reaction rate of $X\left({ }^{4} \mathrm{He}, \gamma\right)^{4} \mathrm{He}_{X}$ increases. The ${ }^{7} \mathrm{Be}$ and ${ }^{7} \mathrm{Li}$ destruction reactions are then not operative [35].

We delineate the parameter region which can be responsible for a reduction of the primordial ${ }^{7} \mathrm{Li}$ abundance. In Figure 4, it is found that the contours of the boundaries for existences of ${ }^{4} \mathrm{He}_{X}^{*}$ are above the contours of the reaction $X\left({ }^{7} \mathrm{Be},{ }^{3} \mathrm{He}\right){ }^{4} \mathrm{He}_{X}$ (the second lowest thin dashed lines). In the parameter region in right upper side from the curve of ${ }^{4} \mathrm{He}_{X}^{*}$, free $X^{0}$ particles are captured onto ${ }^{4} \mathrm{He}$ at $T_{9} \sim 1$ before they can react with ${ }^{7} \mathrm{Be}$ to reduce its abundance.

In the shaded region below that curve and above the curve of $X\left({ }^{7} \mathrm{Be},{ }^{3} \mathrm{He}\right){ }^{4} \mathrm{He} e_{X}$, some amount of free $X^{0}$ s possibly remain, and they can reduce the ${ }^{7} \mathrm{Be}$ abundance. This shaded region is, therefore, a possible parameter region where the ${ }^{7} \mathrm{Li}$ problem is solved. 


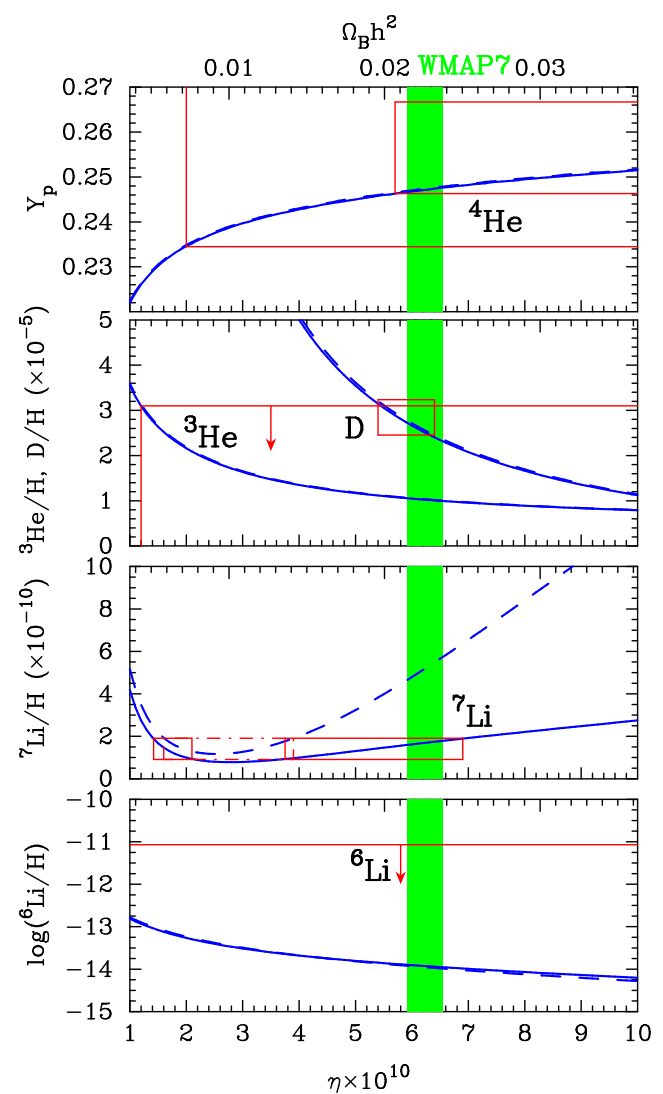

Fig. 3. Abundances of ${ }^{4} \mathrm{He}$ (mass fraction), $\mathrm{D},{ }^{3} \mathrm{He},{ }^{7} \mathrm{Li}$ and ${ }^{6} \mathrm{Li}$ (by number relative to $\mathrm{H}$ ) as a function of the baryon-to-photon ratio $\eta$ or the baryon energy density $\Omega_{B} h^{2}$ of the universe. The solid curves are the calculated results in the $X$ catalyzed BBN for the case of $\left(m_{X}, \delta, Y_{X}, \tau_{X}\right)=\left(100 \mathrm{GeV}, 0.1,1.5 \times 10^{-14}, \infty\right)$, while the dashed curves are those in the standard BBN. The boxes represent the adopted abundance constraints from Refs. [39,40] for ${ }^{4} \mathrm{He}$, [41] for $\mathrm{D}$, [42] for ${ }^{3} \mathrm{He}$, [4] for ${ }^{7} \mathrm{Li}$, and [2] for ${ }^{6} \mathrm{Li}$, respectively. The vertical stripe corresponds to the $2 \sigma \Omega_{B} h^{2}$ limits provided by WMAP [9]. This figure is reprinted from Ref. [35]

\section{Conclusions and discussion}

We have investigated effects of a long-lived strongly interacting massive particle (SIMP) $X^{0}$ on BBN for different masses $m_{X}$ and strengths of $X N$ interaction, i.e., $\delta$. Binding energies of $X$-nuclei (bound states of nuclei and an $X^{0}$ particle) are calculated for two types of $X N$ potentials, i.e., Gaussian and well types [35] although we showed the result of only the Gaussian case in this paper.

We compute the time evolution of light element abundances. We found that ${ }^{7} \mathrm{Be}$ and ${ }^{7} \mathrm{Li}$ can be destroyed by the nuclear capture reactions of free $X^{0}$ particles. The destruction reactions are $X\left({ }^{7} \mathrm{Be},{ }^{3} \mathrm{He}\right){ }^{4} \mathrm{He}_{X}$ and $X\left({ }^{7} \mathrm{Li}, t\right){ }^{4} \mathrm{He}_{X}$. The lack of an excited state of ${ }^{4} \mathrm{He}_{X}$ with a relative angular momentum $L=1$ makes it possible that some fraction of the $X^{0}$ particles escape capture by ${ }^{4} \mathrm{He}$. We suggest that the ${ }^{7} \mathrm{Li}$ problem could be solved based upon a net work calculation of catalyzed BBN, and found the parameter region in the $\left(m_{X}, \delta\right)$ plane where the ${ }^{7} \mathrm{Li}$ problem can be solved.

We note that the results have been derived under the assumption that the $X^{0}$ particles do not affect nuclear structure apart from sticking unaltered nuclei to the particles. This rough approximation is unlikely to be true especially in the case of relatively large interaction strength since the $X N$ potential is not much weaker than the $N N$ potential and can not be neglected. More realistic estimations of wave functions and binding energies of $X$-nuclei including such changes in nuclear structures with the use of three or more-body models are necessary. Although we expect errors in the calculated abundances 


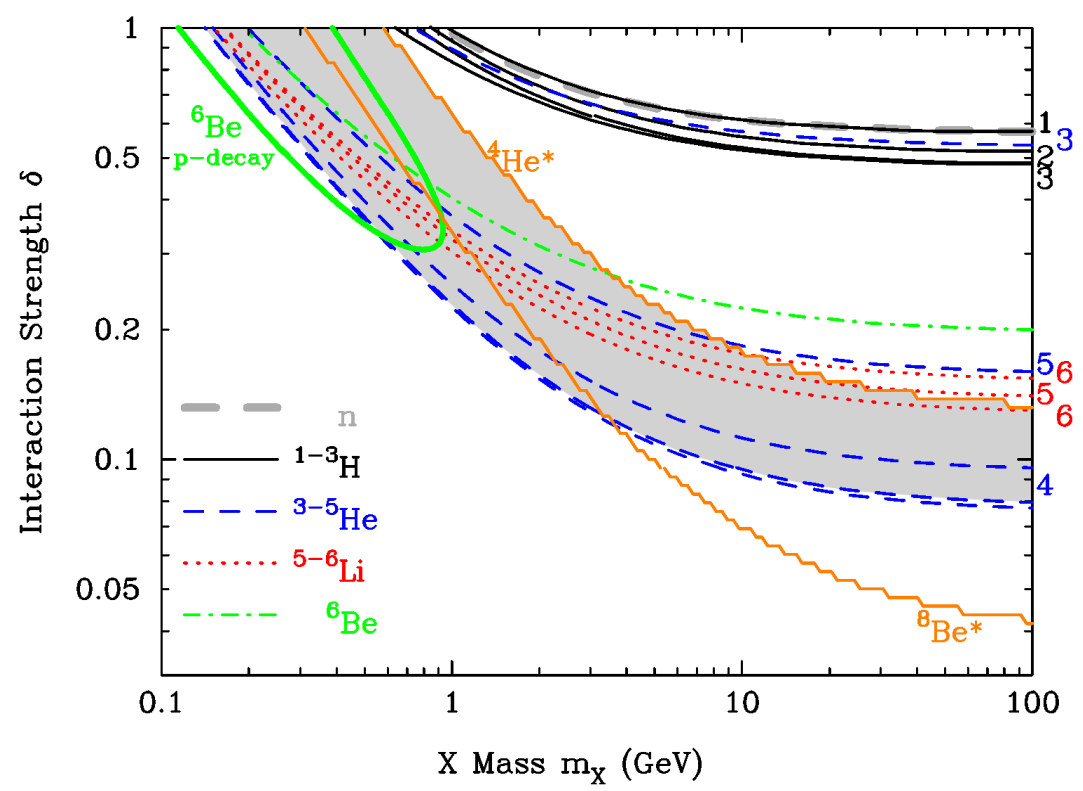

Fig. 4. Contours of binding energies between nuclei and an $X^{0}$ particle corresponding to $Q=0$ of reactions [35] (thin and thick smooth curves). Numbers attached to the contours indicate mass numbers of nuclei. Above the contours, reaction $Q$-values are positive. Zigzag curves correspond to boundaries above which an excited state of ${ }^{4} \mathrm{He}^{*}$ (upper line) and ${ }^{8} \mathrm{Be}^{*}$ (lower) exist, respectively. In the shaded region, the ${ }^{7} \mathrm{Li}$ problem can be resolved. This figure is reprinted from Ref. [35]

in the $X$-catalyzed BBN, which stem from rough approximations we took, of one order of magnitude or so, we suggest that there is a possibility of ${ }^{7} \mathrm{Li}$ reduction in the BBN model including a long-lived sub-SIMP.

\section{Acknowledgment}

This work is supported by Grant-in-Aid for JSPS Fellows No.21.6817 (Kusakabe) and Grant-in-Aid for Scientific Research from the Ministry of Education, Science, Sports, and Culture (MEXT), Japan, No.22540267 and No.21111006 (Kawasaki) and also by World Premier International Research Center Initiative (WPI Initiative), MEXT, Japan.

\section{References}

1. J. Melendez and I. Ramirez. Astrophys. J., 615, (2004) L33

2. M. Asplund, D. L. Lambert, P. E. Nissen, F. Primas, and V. V. Smith. Astrophys. J., 644, (2006) 229-259

3. F. Spite and M. Spite. Astron. Astrophys., 115, (1982) 357-366

4. S. G. Ryan, T. C. Beers, K. A. Olive, B. D. Fields, and J. E. Norris. Astrophys. J., 530, (2000) L57-L60

5. P. Bonifacio, P. Molaro, T. Sivarani, R. Cayrel, M. Spite, F. Spite, B. Plez, J. Andersen, B. Barbuy, T. C. Beers, E. Depagne, V. Hill, P. François, B. Nordström, and F. Primas. Astron. Astrophys., 462, (2007) 851-864

6. J. R. Shi, T. Gehren, H. W. Zhang, J. L. Zeng, and G. Zhao. Astron. Astrophys., 465, (2007) 587-591

7. W. Aoki et al. Astrophys. J., 698, (2009) 1803-1812 
8. R. H. Cyburt, B. D. Fields, and K. A. Olive. JCAP, 0811, (2008) 012

9. D. Larson et al. Astrophys. J. Suppl., 192, (2011) 16

10. O. Richard, G. Michaud, and J. Richer. Astrophys. J., 619, (2005) 538-548

11. A. J. Korn et al. Nature, 442, (2006) 657-659

12. K. Lind, F. Primas, C. Charbonnel, F. Grundahl, and M. Asplund. Astron. Astrophys., 503, (2009) 545-557

13. N. Arkani-Hamed and S. Dimopoulos. JHEP, 06, (2005) 073

14. N. Arkani-Hamed, S. Dimopoulos, G. F. Giudice, and A. Romanino. Nucl. Phys., B709, (2005) 3-46

15. S. Raby. Phys. Lett., B422, (1998) 158-162

16. S. Shirai, M. Yamazaki, and K. Yonekura. JHEP, 06, (2010) 056

17. L. Covi, M. Olechowski, S. Pokorski, K. Turzynski, and J. D. Wells. JHEP 1101, (2011) 033

18. U. Sarid and S. D. Thomas. Phys. Rev. Lett., 85, (2000) 1178-1181

19. J. Hisano, K. Nakayama, S. Sugiyama, T. Takesako, and M. Yamanaka. Phys. Lett., B691, (2010) $46-55$

20. K. Nakayama, F. Takahashi, and T. T. Yanagida. Phys. Lett., B697, (2011) 275

21. J. Kang, M. A. Luty, and S. Nasri. JHEP, 09, (2008) 086

22. E. W. Kolb and M. S. Turner. The early universe (Addison-Wesley, 1990) p64

23. S. Wolfram. Phys. Lett., B82, (1979) 65

24. C. B. Dover, T. K. Gaisser, and G. Steigman. Phys. Rev. Lett., 42, (1979) 1117

25. G. D. Starkman, A. Gould, R. Esmailzadeh, and S. Dimopoulos. Phys. Rev., D41, (1990) 3594

26. D. A. Dicus and V. L. Teplitz. Phys. Rev. Lett., 44(3), (1980) 218-221

27. R. N. Mohapatra and V. L. Teplitz. Phys. Rev. Lett., 81, (1998) 3079-3082

28. K. Nakamura and Particle Data Group. Journal of Physics G Nuclear Physics, 37(7), (2010) 075021

29. M. Kusakabe, T. Kajino, T. Yoshida, and G. J. Mathews. Phys. Rev., D80, (2009) 103501

30. N. Prantzos, M. Casse, and E. Vangioni-Flam. Astrophys. J., 403, (1993) 630-643

31. R. Ramaty, B. Kozlovsky, R. E. Lingenfelter, and H. Reeves. Astrophys. J., 488, (1997) 730-748

32. M. Kusakabe. Astrophys. J., 681, (2008) 18-26

33. S. E. Woosley and T. A. Weaver. Astrophys. J. Suppl., 101, (1995) 181-235

34. T. Yoshida, T. Kajino, and D. H. Hartmann. Phys. Rev. Lett., 94, (2005) 231101

35. M. Kawasaki and M. Kusakabe, Phys. Rev. D 83, (2011) 055011

36. E. Hiyama, this volume

37. E. Hiyama, M. Kamimura, T. Motoba, T. Yamada, and Y. Yamamoto. Phys. Rev., C66, (2002) 024007

38. E. Hiyama, Y. Kino, and M. Kamimura. Prog. Part. Nucl. Phys., 51, (2003) 223-307

39. Y. I. Izotov and T. X. Thuan. Astrophys. J., 710, (2010):L67-L71

40. E. Aver, K. A. Olive, and E. D. Skillman. JCAP, 1005, (2010) 003

41. M. Pettini, B. J. Zych, M. T. Murphy, A. Lewis, and C. C. Steidel. MNRAS, 391, (2008) 14991510

42. T. M. Bania, Robert T. Rood, and Dana S. Balser. Nature, 415, (2002) 54-57 